\title{
The procerebrum is necessary for odor-aversion learning in the terrestrial slug Limax valentianus
}

\author{
Yoko Kasai, ${ }^{1}$ Satoshi Watanabe, ${ }^{1}$ Yutaka Kirino, ${ }^{1}$ and Ryota Matsuo ${ }^{1,2,3}$ \\ ${ }^{1}$ Laboratory of Neurobiophysics, School of Pharmaceutical Sciences, The University of Tokyo, Bunkyo-ku, Tokyo 113-0033, Japan; \\ ${ }^{2}$ Laboratory of Functional Biology, Faculty of Pharmaceutical Sciences at Kagawa Campus, Tokushima Bunri University, Shido, \\ Sanuki, Kagawa 769-2193, Japan
}

\begin{abstract}
The terrestrial slug Limax has a highly developed ability to associate the odor of some foods (e.g., carrot juice) with aversive stimuli such as the bitter taste of quinidine solution. The procerebrum (PC) is a part of the slug's brain thought to be involved in odor-aversion learning, but direct evidence is still lacking. Here we present evidence showing that the PC is essential for odor-aversion learning. Unlike sham-operated slugs, PC ablation $7 \mathrm{~d}$ prior to conditioning showed that most slugs did not avoid carrot juice in the memory retention test conducted $24 \mathrm{~h}$ after the conditioning. Slugs with the PC ablated $3 \mathrm{~h}, 1 \mathrm{~d}, 3 \mathrm{~d}$, or $7 \mathrm{~d}$ after conditioning and examined by the memory retention test at $3 \mathrm{~d}$ after the PC ablation were also less likely to avoid carrot juice than sham-operated slugs. The PC ablation did not damage the ability of the slugs to sense attractive odor (everyday food) or innately aversive odor (onion or garlic). These results demonstrate that the PC is a necessary component in the retention and/or retrieval of odor-aversion memory.
\end{abstract}

The neuronal mechanism of olfactory learning has been largely investigated in mammals and insects, and the information processing of olfactory sensing in the olfactory centers has been well studied in these animal models (Davis 2004). However, there are several limitations in the use of these animals for the study of the neuronal mechanism of olfactory learning, such as the large number of neurons involved and the difficulty of identifying output motor pathways. The terrestrial mollusk is a suitable animal model because in vitro study of olfaction and learning is possible using an isolated whole brain, which usually has far fewer neurons $\left(\sim 10^{5}\right.$ for Limax) with relatively low complexity (Chang and Gelperin 1980; Gervais et al. 1996; Teyke and Gelperin 1999; Inoue et al. 2004, 2006). These characteristics provide incomparable advantages to electrophysiological and histological studies. In particular, the terrestrial slug Limax has a highly developed ability to acquire an aversive response to the odor of some foods (e.g., carrot) once it is presented in combination with an aversive stimulus (e.g., application of $\mathrm{CO}_{2}$ gas or quinidine sulfate solution) (Gelperin 1975; Sahley et al. 1981). This type of associative memory is easily formed by a single conditioning and persists for several weeks.

The procerebrum (PC), a part of the cerebral ganglion in the central nervous system (CNS) of Limax, is thought to be one of the loci of olfactory information processing. Histological studies have shown that the PC is situated anatomically where it can receive not only indirect input from the olfactory epithelium (tip of a tentacle) via tentacle ganglion but also direct input from the olfactory epithelium (Chase and Tolloczko 1989; Kimura et al. 1998a; M. Fukumoto, M. Sakura, I. Ito, S. Watanabe, and Y. Kirino, unpubl.). Electrophysiological studies have demonstrated that the synchronous oscillation of the local field potential (LFP) recorded in the tentacle ganglion and the $\mathrm{PC}$ is responsive to the olfactory inputs to the olfactory epithelium (Gelperin and Tank 1990; Ito et al. 2001). These features are analogous to those of the olfactory bulb in mammals and the antennal lobe in insects (Gel-

${ }^{3}$ Corresponding author.

E-mail matsuor@kph.bunri-u.ac.jp; fax +81-87-894-0181.

Article published online before print. Article and publication date are at http:// www.learnmem.org/cgi/doi/10.1101/lm.257606. perin 1999). It has also been demonstrated that the synchronous oscillation of the PC activity is involved in odor discrimination (Teyke and Gelperin 1999). Moreover, a series of studies has indirectly supported the notion that the PC is implicated in the formation or storage of olfactory memory. Firstly, changes in the frequency of LFP oscillation depend on the acquired meaning of the odor: Aversively conditioned odor evokes lower frequency oscillation, while appetitively conditioned odor increases the oscillatory frequency of LFP in the PC (Kimura et al. 1998b). Secondly, post-conditioning injection of Lucifer Yellow specifically labeled the PC neurons only when the unconditioned and conditioned stimuli were presented simultaneously (Kimura et al. 1998a; Ermentrout et al. 2001). Thirdly, the mRNA of LAPS18, which encodes secreted protein, was up-regulated in the PC $3 \mathrm{~h}$ after olfactory conditioning (Nakaya et al. 2001). However, while these studies showed the correlation between the physiological/ biochemical activities of the PC and olfactory learning, none of these studies clearly demonstrated that the PC is necessary for olfactory learning.

The present study was designed to define the role of the PC in olfactory learning using a lesion experiment. We developed the method for the PC ablation in anaesthetized slugs with a high recovery rate. In the first experiment, the PC ablation was performed before olfactory conditioning, and the recovered slugs were conditioned and tested for memory retention. In the second experiment, the PC was ablated various intervals after the olfactory conditioning, and memory retention was tested after recovery. We also examined whether the PC ablation left the olfactory sensibility and the mobility of the slug.

\section{Results}

Experiment I: Pre-conditioning ablation of the PC impaired odor-aversion memory

To investigate the role of the PC in odor-aversion memory in Limax, the PC was ablated bilaterally $7 \mathrm{~d}$ prior to conditioning (Fig. 1A and 1B). As shown in Figure 2A, 36 PC-ablated slugs and 37 sham-operated slugs were conditioned, and the memory retention test was performed the next day in a blind manner. For the sham-operated group of slugs, 34 (91.9\%) out of 37 slugs 

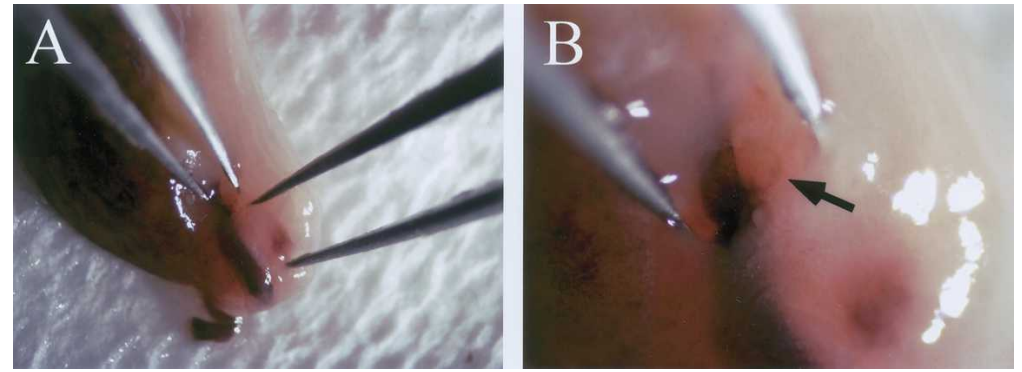

Figure 1. Photograph of the $P C$ ablation surgery. $(A)$ An anesthetized slug was laid on a paper towel, and the side of the head was cut. (B) Magnified view of the cut site. The arrow indicates the left side of the PC.

avoided the carrot juice for $3 \mathrm{~min}$, while only six (16.7\%) out of 36 slugs in the PC-ablated groups did (Fig. 2B). This difference was significant $\left(\chi^{2}=41.7, P<0.001\right.$, d.f. $\left.=1\right)$.

Odor sensibility was indistinguishable between the PCablated group and the sham-operated group as judged by the number of successes in the approach to the diluted powder mixture laid in either the right or the left quadrant within $3 \mathrm{~min}$ (Table $1 ; \chi^{2}=0.188, P=0.665$, d.f. $=3$; see Materials and Methods for details). The mobility of the slugs was slightly affected in the PC-ablated group; the time it took to reach the diluted powder mixture was $48.3 \pm 13.6 \mathrm{sec}$ for the PC-ablated group (the total number of successful approaches $=169$ ) while for the shamoperated group (the total number of successful approaches $=$ 173), the mean time taken was $40.0 \pm 12.1 \mathrm{sec}(P<0.01$, twotailed $t$-test). However, there was no difference in mobility within the PC-ablated group between the slugs that reached the carrot juice within $3 \mathrm{~min}$ and those that did not $(47.5 \pm 13.9 \mathrm{sec}$; $n=30$ and $52.4 \pm 12.5 \mathrm{sec} ; n=6$, respectively; $P=0.418$, twotailed $t$-test). There was also no difference in the mobility within the sham-operated group $(45.2 \pm 18.2 \mathrm{sec} ; n=3$ and $39.5 \pm 11.7$ sec; $n=34$, respectively, $P=0.645$, two-tailed $t$-test). The ability

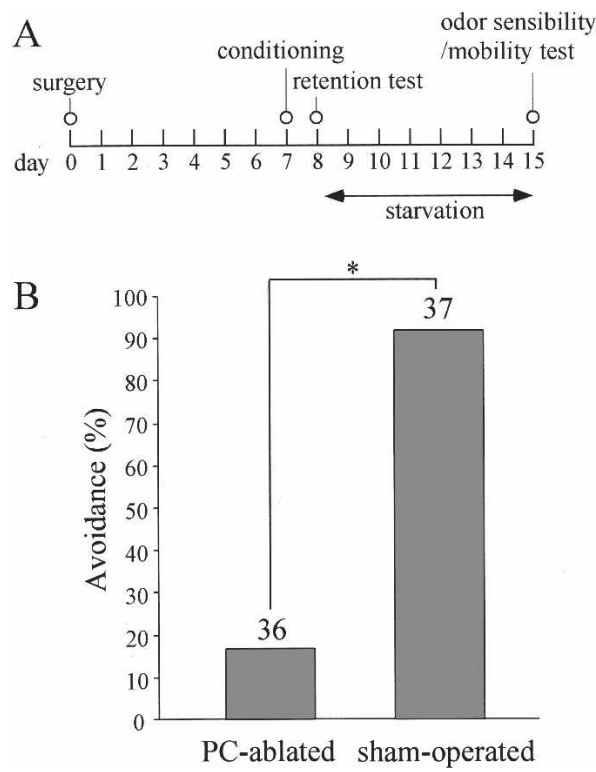

Figure 2. Experiment I: Pre-conditioning PC ablation impaired odoraversion learning. (A) Experimental schedule. (B) The avoidance rate (\%) was substantially reduced in the PC-ablated group in comparison to the sham-operated groups on the day after the conditioning $\left(P<0.001\right.$ by $\chi^{2}$ test). The numerals above the columns indicate the number of slugs used for the conditioning. to avoid innately aversive odor was also not affected by the PC ablation because no slugs in either group reached the garlic homogenate within $3 \mathrm{~min}(n=73)$ laid in a $180^{\circ}$ arc with a $9-\mathrm{cm}$ radius.

To further confirm the ability of the slugs to sense and direct their movement after a short recovery period ( $8 \mathrm{~d}$ after surgery), we monitored their behavior on a glass plate when they were surrounded by a circle (5-cm radius) consist of a favorable odorant (humidified everyday food) on one half and an aversive odorant (onion or garlic homogenates, Inoue et al. 2004, 2006) on the other. Almost all the slugs (seven out of seven sham-operated slugs, seven out of eight PC-ablated slugs) successfully reached the everyday food (upper half) without touching the repellent odor sources (Fig. 3A,B), showing the intact ability to sense and regulate their crawling as early as $8 \mathrm{~d}$ after the surgery.

To confirm the successful ablation of the PC, we randomly chose a sample of slugs that had avoided the carrot juice (five slugs) and those that had not avoided the carrot juice (11 slugs) in the PC-ablation group, and analyzed the residual PC region histologically. This analysis confirmed the successful ablation of the bilateral PC for almost all the slugs we examined (Fig. 4A,B). For comparison, a stained section derived from a naive slug was also shown (Fig. 4C). We also measured the areas of the residual $\mathrm{PC}$ region in these stained sections to investigate the relation between the extent of the lesion of the PC and the learning behavior. But no significant difference was found in the residual PC areas between the slugs that reached the carrot juice within 3 min and those that did not (Fig. 5), although there was a tendency toward a reduced residual PC area in the slugs that reached the carrot juice within $3 \mathrm{~min}\left(1.07 \times 10^{-2} \pm 0.89 \times 10^{-2} \mathrm{~mm}^{2}\right.$ $[n=11]$ and $1.43 \times 10^{-2} \pm 0.93 \times 10^{-2} \mathrm{~mm}^{2}[n=5]$, respectively; $P=0.510$, two-tailed $t$-test).

\section{Experiment II: Post-conditioning PC ablation impaired storage or retrieval of odor-aversion memory}

In the second experiment, the PC was ablated after conditioning. The PC was ablated $3 \mathrm{~h}, 1 \mathrm{~d}, 3 \mathrm{~d}$, or $7 \mathrm{~d}$ after conditioning, and the memory retention test was performed $3 \mathrm{~d}$ after the surgery (Fig. 6A). In this experiment, we prepared not only PC-ablated and sham-operated slugs, but also unoperated slugs conditioned according to the same time schedule. The survival rate at $3 \mathrm{~d}$ after the surgery was $66.2 \%$ (88 slugs out of 133 ) in the PC ablation group. For $5.7 \%$ of the survived slugs (five slugs out of 88 ), a mobility defect was detected, such as an inability to move in a straight line, and they always circled on the same spot in a constant turning direction. These slugs were not used for further experiments.

The PC-ablated slugs showed reduced avoidance rates in all the groups with different time intervals between conditioning

Table 1. Distribution of the number of successful approaches to the diluted powder mixture of the PC-ablated and the sham-operated slugs

\begin{tabular}{lccccc}
\hline & $\mathbf{5}$ times & $\mathbf{4}$ times & $\mathbf{3}$ times & $\mathbf{0 - 2}$ times & Total \\
\hline PC-ablated & 26 & 9 & 1 & 0 & 36 \\
Sham-operated & 25 & 12 & 0 & 0 & 37 \\
\hline
\end{tabular}

There was no significant difference $\left(P=0.665\right.$, by $\chi^{2}$ test). 
A
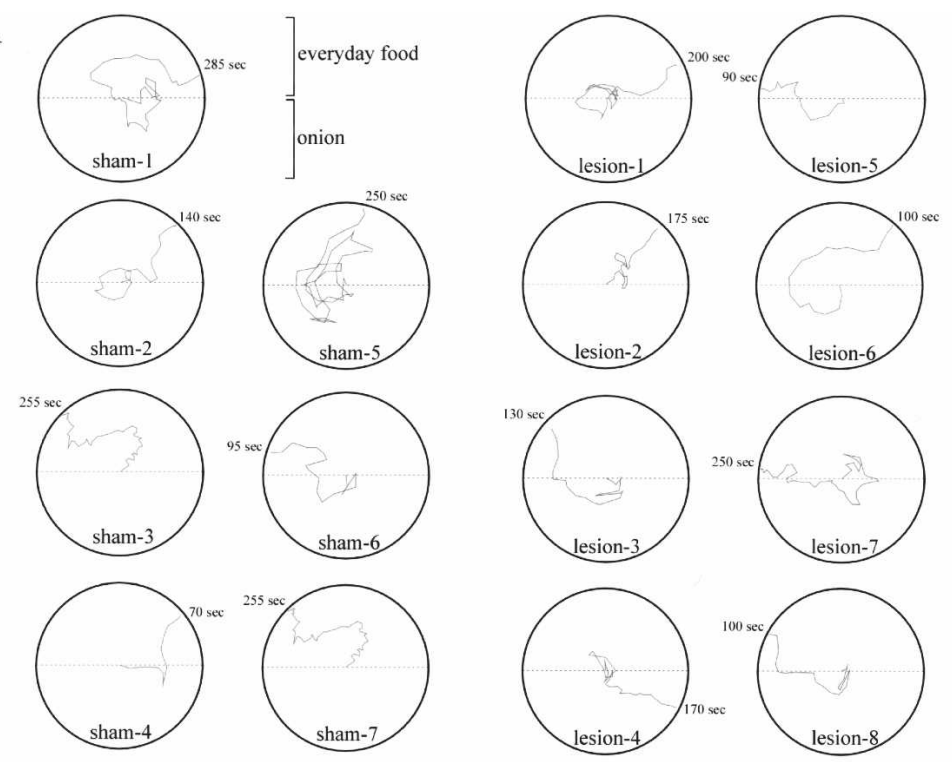

Sham

B
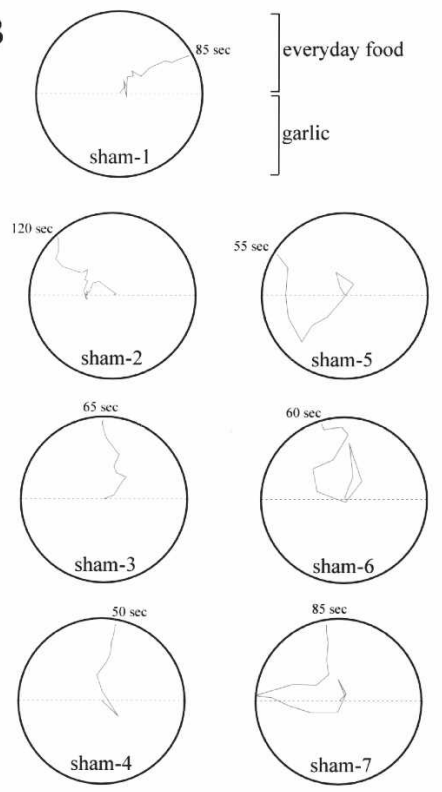

Sham

\section{PC-ablated}
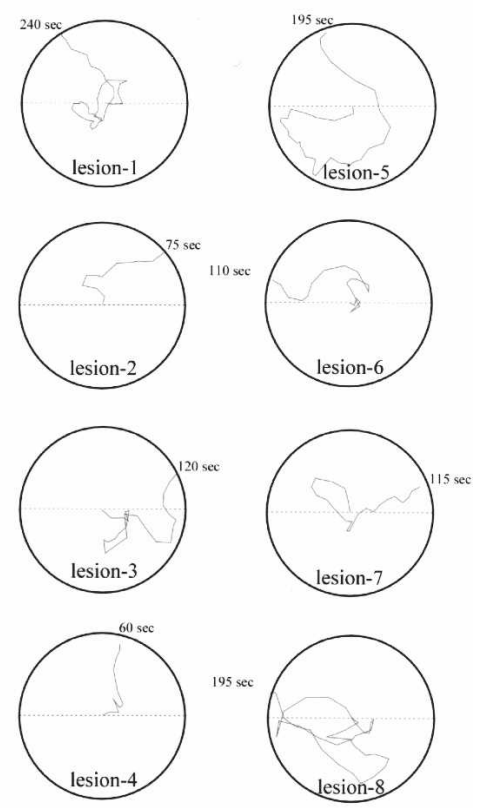

PC-ablated

Figure 3. Tracings of the PC-ablated and sham-operated slugs $8 \mathrm{~d}$ after surgery. The humidified everyday food was placed on the upper half of each circle, and repellent odorant ([A] onion or $[B]$ garlic homogenates) was placed on the lower half. The time the slug reached either odor source is indicated.

and surgery in comparison to sham-operated slugs $\left(\chi^{2}=5.81\right.$, $P<0.05$ for a 3 -h interval; $\chi^{2}=5.16, P<0.05$ for a 1 -d interval; $\chi^{2}=4.25, P<0.05$ for a 3 -d interval; $\chi^{2}=9.25, P<0.01$ for a 7 -d interval, Fig. 6B). There was no difference in the avoidance rates between sham-operated and unoperated slugs $(P=0.0882$ for a 3-h interval; $P=0.915$ for a 1 -d interval; $P=0.474$ for a 3 -d interval; $P=0.329$ for a $7-d$ interval, Fig. $6 \mathrm{~B}$ ).

After the memory retention test, the mobility test was performed on the same day using the diluted powder mixture as an attractive odor source in order to examine the extent of recovery of the PC-ablated slugs from the surgery. Here again, the PCablated slugs took longer to get to the powder mixture than sham-operated slugs, although all the slugs reached the powder mixture within $3 \mathrm{~min}$. All the data from the PCablated slugs and sham-operated slugs were averaged $(53.7 \pm 25.2 \mathrm{sec} ; n=73$ and $44.9 \pm 19.1 \mathrm{sec} ; n=63$, respectively; $P<0.05$, two-tailed $t$-test). There was no difference in the mobility in the PC-ablated group between the slugs that reached the carrot juice within $3 \mathrm{~min}$ and those that did not, although the slug that did not reach the carrot juice within $3 \mathrm{~min}$ took slightly longer to reach the diluted powder mixture, but the difference was not significant $(47.5 \pm 16.3 \mathrm{sec} ; n=24$ and $56.7 \pm 28.2$ sec; $n=49$, respectively; $P=0.083$, twotailed $t$-test). In the sham-operated groups, there was also no difference in mobility between the slugs that reached the carrot juice within $3 \mathrm{~min}$ and those that did not, but, again, the slugs that did not reach the carrot juice within 3 min tended to take longer to reach the powder mixture, but the difference was not significant $(47.3 \pm 20.3 \mathrm{sec} ; n=46$ and $38.5 \pm 14.0 \mathrm{sec} ; n=17$, respectively; $P=0.059$, two-tailed $t$-test).

\section{Discussion}

In Drosophila, chemical and genetic ablation of mushroom bodies abolished associative olfactory learning without affecting most of the sensorimotor abilities (de Belle and Heisenberg 1994; Dubnau et al. 2001; McGuire et al. 2001). In rats, olfactory fear learning is damaged by excitotoxic lesions of basolateral amygdala or anterior perirhinal cortex (Otto et al. 2000). However, there are only a few cases in which the ability to achieve associative olfactory learning is attributable to one or a few neuronal structures. In this study, we demonstrated that the PC of the Limax is necessary for associative olfactory learning.

In Experiment I, we demonstrated that the pre-conditioning ablation of the PC substantially reduced the avoidance rate from the conditioned odor of carrot juice $1 \mathrm{~d}$ after the conditioning, suggesting that the PC is necessary for any of the temporal stages of memory: acquisition, storage, or retrieval. Odor sensibility/mobility tests demonstrated the intact ability to avert the crawling direction from the repellent odorant and to approach the attractive odorant, in spite of the fact that some slugs sometimes showed insufficient extension of one or both of their superior tentacles (not shown). The video recording further confirmed their sound sensorimotor ability $8 \mathrm{~d}$ after the surgery, corresponding to the time when the retention test was performed. In Experiment II, we reduced the number of possible damaged stages by ablating the PC after the conditioning. The fact that the post-conditioning ablation of the $\mathrm{PC}$ reduced the avoidance rate from the conditioned odor suggests that the PC is involved at least in either memory storage or 
A
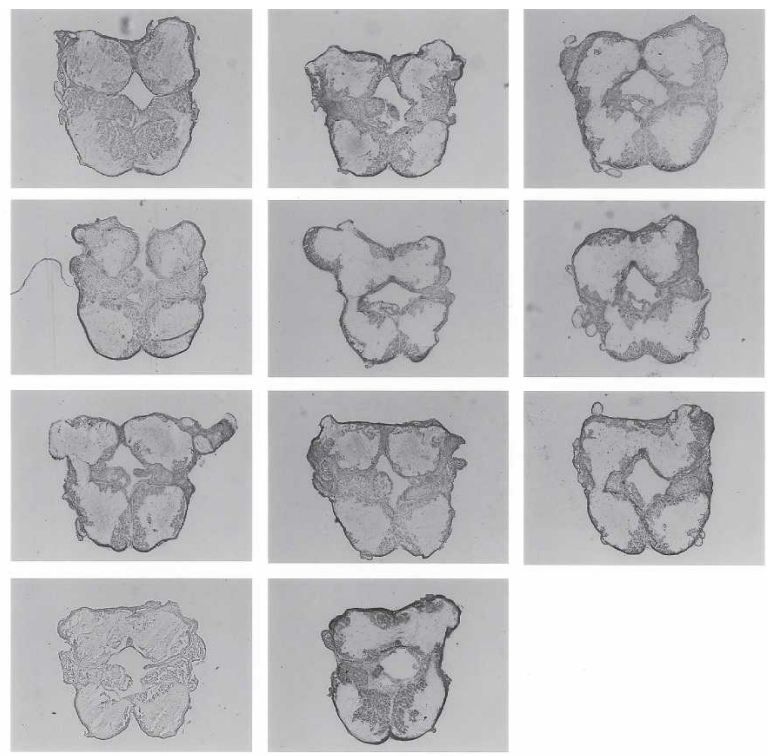

B
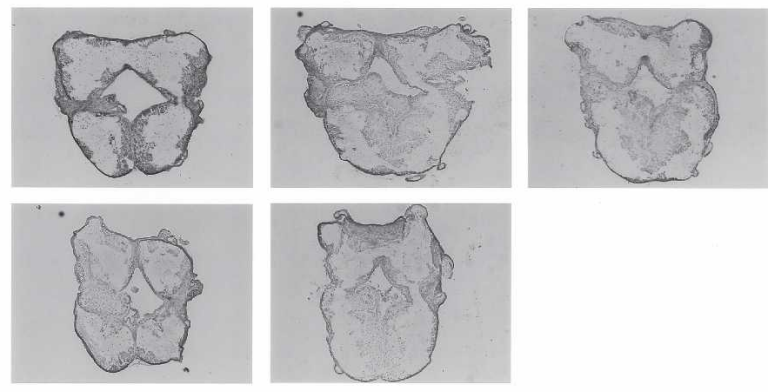

C

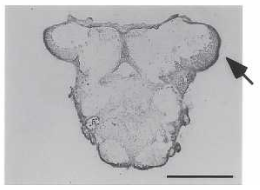

Figure 4. Histological analysis of the $\mathrm{PC}$-ablated brain of the slugs. Toluidine blue-stained sections of the brains of $(A) 11$ randomly chosen slugs that had not avoided the conditioned odor of the carrot juice and $(B)$ five randomly chosen slugs that avoided the conditioned odor of the carrot juice. (C) A section of the brain from a naive slug. The arrow indicates the PC. Scale bar, $500 \mu \mathrm{m}$.

retrieval. If the PC is the storage site of olfactory memory, it holds memory for $7 \mathrm{~d}$ at least and does not transfer the engram to the other sites of the brain, because the odor-aversion memory was damaged by ablation of the PC even $7 \mathrm{~d}$ after conditioning (Fig. $6 \mathrm{~B})$. It is not possible to determine precisely whether the PC functions for either or both of the memory storage and retrieval stages. This is because the lesion experiment is irreversible, and techniques such as temporally regulative genetic interference and reversible pharmacological inhibition of the PC are not yet available for slugs, although they have been used in Drosophila (Dubnau et al. 2001; McGuire et al. 2001) and mammals (Mayford et al. 1996; Izquierdo et al. 1997).

There was a greater tendency for the PC-ablated slugs to avoid the carrot odor in the memory retention test in Experiment II than in Experiment I (Figs. 2B, 6B). This could be due to several slugs in Experiment II that did not recover completely because of the short recovery period ( $3 \mathrm{~d})$ following the surgery. These slugs may have failed to reach the carrot juice within 3 min even in the absence of aversive memory. This might cause overestimation of the number of memory-intact slugs. In fact, we found a tendency for the memory-lost slugs to move toward powder mixture more slowly than the memory-intact slugs in Experiment II, although the difference was not statistically significant. Another possibility is that the $\mathrm{PC}$ is more exclusively involved in the acquisition of memory than in storage or retrieval, and some other part of the CNS might have some ability to compensate these later memory stages.

We did not find any correlation between the extent of the PC lesion and the deficiency in memory, although there was a tendency toward a reduced area of the residual PC in the memory-intact groups (Fig. 5). It is not yet clear whether the remaining PC could play a role in odor-aversion memory since the number of slugs investigated was small, especially for the memory-intact slugs $(n=5)$. A larger scale of investigation is required to test this possibility.

In spite of a series of studies implying the involvement of the PC in the odor-aversion learning in Limax (Kimura et al. 1998a,b; Ermentrout et al. 2001; Nakaya et al. 2001), there is no decisive evidence that the PC plays an essential role, possibly because the surgery was technically difficult. In this study, we developed a less invasive surgical procedure to remove both sides of the PC from the slug with a relatively high recovery rate, making it possible to study the behavior of recovered slugs. Although the PC-ablated slugs tended to move slowly, the conclusions of this study were not affected because most of the PCablated slugs reached the carrot juice within $3 \mathrm{~min}$ in spite of their slowness.

The PC has long been believed to be the locus involved not only in odor-aversion memory but also in discrimination between similar odors (Teyke and Gelperin 1999). It is logically difficult, however, to examine whether the PC is also necessary for such odor discrimination by the PC ablation experiment, because, in many cases, the discrimination task depends on the ability of the slug to acquire and retrieve the odor-aversion memory (Sakura et al. 2004). Therefore, the possibility remains open that the PC plays a role in odor discrimination. In fact, it has been suggested that the information about odor identity is

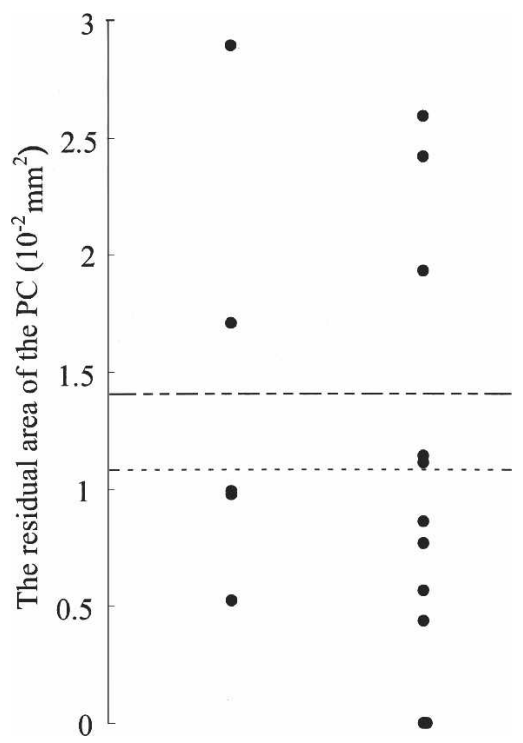

Figure 5. There was no significant difference in the residual area of the PC between the slugs that reached the carrot juice within $3 \min (n=5$, left dots) and those that did not ( $n=11$, right dots). The average areas $\left(1.42 \pm 0.92 \times 10^{-2} \mathrm{~mm}^{2}\right.$ and $\left.1.07 \pm 0.89 \times 10^{-2} \mathrm{~mm}^{2}\right)$ are indicated as broken and dotted lines, respectively. 
A
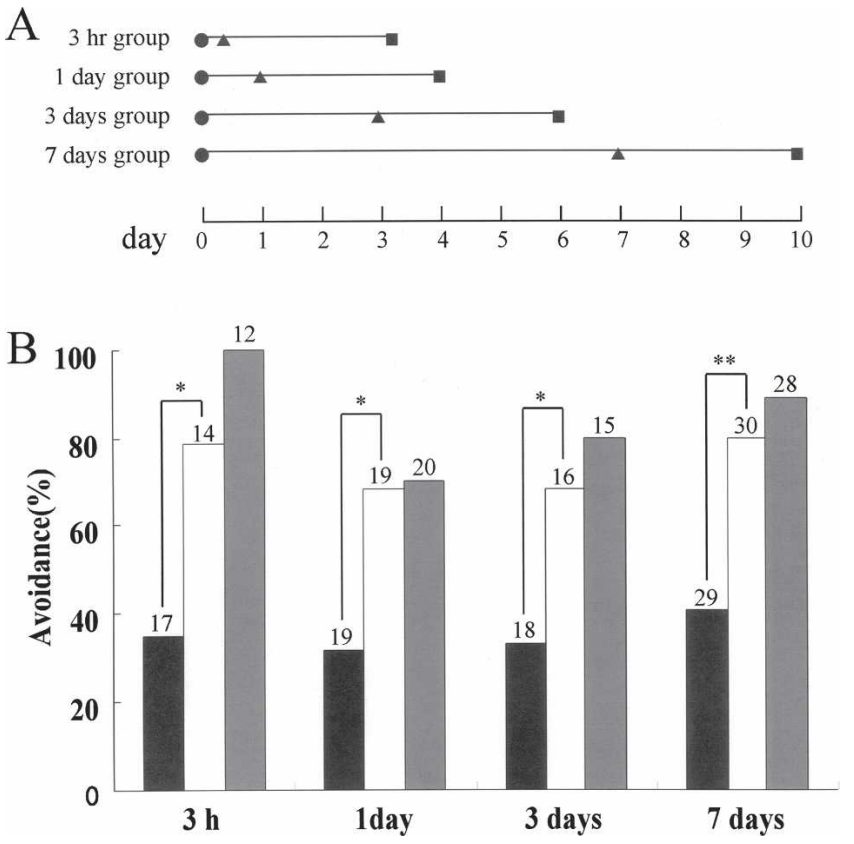

Figure 6. Experiment II: The post-conditioning PC-ablation impaired the odor-aversion learning. (A) The experimental schedule. The conditioning, the surgery, and the retention test are indicated by $\boldsymbol{\bullet}, \mathbf{\Lambda}$, and $\mathbf{\square}$, respectively. (B) The avoidance rate (\%) was substantially reduced in the PC-ablated group than in the sham-operated groups irrespective of the interval between the conditioning and the surgery. The numerals above the columns indicate the number of slugs used for the conditioning. ${ }^{*} P<0.05$ and ${ }^{* *} P<0.01$ by $\chi^{2}$ test. Solid bars: PC-ablated slugs; open bars: sham-operated slugs; shaded bars: naive slugs.

encoded by the activity of nonbursting neurons in the PC (Murakami et al. 2003).

The PC-ablated slugs moved normally toward their everyday food laid in front of them to the right or left side, selecting appropriate direction, and their success rate was not statistically different from that of sham-operated slugs. The PC-ablated slugs also retreated from onion and garlic homogenates, which has an odor to which slugs are innately averse (Sahley 1990; Matsuo et al. 2002; Inoue et al. 2004, 2006). Taken together, it seems that the PC-ablated slugs retained the ability to sense and approach innately motivated odorants, and to detect and retreat from innately aversive odorants. Since the PC is often considered an odor information-processing center, sometimes analogized with the olfactory bulb of mammals (Gelperin 1999), how can slugs sense odors without the PC? In Limax, olfactory afferent inputs from the olfactory epithelium are conveyed through the tentacular nerves (in a superior tentacle) and the branch of medial lip nerves (in an inferior tentacle). Kimura et al. (1998a) have reported that both of these nerves have projections not only to the ipsilateral PC but also to the metacerebral lobe. It is also known that there exist at least two kinds of afferent pathways from a tentacle in the giant snail Achatina fulica: One makes contacts with neuropiles of the PC neurons, and the other bypasses the PC, making direct contacts with extended dendrites of pedal neurons (Chase and Tolloczko 1989, 1993). Such direct pathways may still be intact after the PC ablation, enabling the slug to sense the odors. In this case, tentacle ganglia or pedal ganglia are candidates for the odor information-processing and/or decisionmaking centers to determine the direction of the slug's locomotion when the olfactory epithelium binds innately aversive or attractive odorants. With a fully intact brain, the PC might modulate such decision making based on the memory stored in it.
Strictly speaking, it could be interpreted that the preference for the odor of everyday food was also acquired and formed by learning as an appetitive memory. One possible explanation to the fact that this memory was not damaged by the PC ablation is that the PC is involved in the aversive learning but not in the appetitive learning. In fact, a differential dependence on neurotransmitters has been reported between aversive and appetitive learning: Aversive associative conditioning depends on serotonin in Helix (Balaban et al. 1987) and Aplysia (Hawkins et al. 1993) while appetitive food attraction conditioning does not in Helix (Teyke 1996). Another explanation is that this memory has been consolidated so firmly by repeated exposure to the powdered mixture from birth, that the PC is no longer required to modulate the slug's behavior. According to the latter interpretation, further investigation is needed to determine the locus where such fully consolidated memories are stored.

\section{Materials and Methods}

\section{Animals}

Terrestrial slugs, Limax valentianus (12-20 wk post-hatching), were maintained in our laboratory at $19^{\circ} \mathrm{C}$. They were fed only on a diet of a humidified powder mixture (for composition, see Nakaya et al. 2001) and had never eaten carrot.

\section{PC ablation}

All the solutions injected into the slugs were sterilized by filtrating through a syringe filter (Corning) immediately before injection. The slugs were anesthetized by injection of $\sim 500 \mu \mathrm{L}$ of icecold $\mathrm{Mg}^{2+}$ buffer $\left(57.6 \mathrm{mM} \mathrm{MgCl}_{2}, 5 \mathrm{mM}\right.$ glucose, $2.36 \mathrm{mM} \mathrm{N}-2$ Hydroxyethylpiperazine- $\mathrm{N}^{\prime}$-2-ethanesulfonic acid [HEPES], 2.64 $\mathrm{mM}$ HEPES-Na) into the body cavity. For the PC ablation group of slugs, small cuts were made with microscissors on both sides of the head, and the PC was exposed and removed bilaterally using sharp microforceps as shown in Figure 1A,B. After surgery, 300 $\mu \mathrm{L}$ of physiological buffer solution $(70 \mathrm{mM} \mathrm{NaCl}, 2 \mathrm{mM} \mathrm{KCl}, 4.7$ $\mathrm{mM} \mathrm{MgCl}_{2}, 4.9 \mathrm{mM} \mathrm{CaCl}_{2}, 5 \mathrm{mM}$ glucose, $2.36 \mathrm{mM}$ HEPES, 2.64 $\mathrm{mM}$ HEPES-Na) were injected into the body cavity to facilitate the recovery from anesthesia. In most cases, the wound was healed spontaneously without any treatment. For the shamoperated group of slugs, small cuts were made on both sides of the head, and the PC was transiently exposed, and then the physiological buffer solution was injected into the body cavity to facilitate the recovery from anesthesia. After surgery, each slug was transferred to a plastic container and maintained individually with free access to the humidified powder mixture until a memory retention test was performed. The survival rate was monitored for the PC ablation group of slugs only in Experiment II.

\section{Associative conditioning and memory retention test}

Conditioning and memory retention test were performed essentially as described previously (Matsuo et al. 2002) using carrot juice and 1\% quinidine sulfate solution as conditioned and unconditioned stimuli, respectively, except that the slugs were not starved before the conditioning. In Experiment I, the conditioning was performed $7 \mathrm{~d}$ after the PC ablation, and the memory retention test took place the next day. Avoidance (\%) was defined as a relative number of slugs that did not reach the carrot juice within $3 \mathrm{~min}$. After the memory retention test, the slugs were starved for $7 \mathrm{~d}$ and the odor sensibility/mobility tests (see below) were performed using the humidified powder mixture and the garlic homogenate. In Experiment II, the PC ablation was performed $3 \mathrm{~h}, 1 \mathrm{~d}, 3 \mathrm{~d}$, or $7 \mathrm{~d}$ after the conditioning, and the memory retention test took place after $3 \mathrm{~d}$ of recovery. Following the memory retention test, the odor sensibility/mobility tests were performed in the same day using only the diluted powder mixture. All tests were performed in a blind manner in which the experimenter was not informed whether each slug was from the PC ablation group or the control group. 


\section{Odor sensibility/mobility test}

The odor sensibility/mobility test was performed as follows in order to assess the effects of the PC ablation on the ability of the slug to sense the odor and to move normally. The experimental chamber was the same as that used for conditioning and for the memory retention test. In Experiment I, $\sim 1 \mathrm{~mL}$ of the diluted humidified powder mixture was laid to the right or left side of the slug in a quarter-circle arc $\left(90^{\circ}\right)$ with a $6-\mathrm{cm}$ radius from the marked point on the glass plate. The slug was placed just behind the marked point and was timed to assess whether it reached the powder mixture within $3 \mathrm{~min}$ after the middle of its body passed the marked point. The slug was gently picked up with forceps just before it touched the powder mixture and was returned to the plastic container. The time it took to reach the powder mixture was also recorded. This procedure was repeated five times for each slug, alternating the side on which the powder mixture was laid to reduce the probability that the slug could reach it by chance. Immediately after these tests, another odor sensibility test was performed using garlic homogenate as an innately aversive odor (Sahley 1990; Matsuo et al. 2002). Garlic homogenate was laid in a $180^{\circ}$ arc with a $9-\mathrm{cm}$ radius from the marked point, and the slug was timed to assess whether it reached the garlic homogenate within $3 \mathrm{~min}$ after passing the marked point. In Experiment II, the mobility test was performed for randomly selected slugs from the PC-ablated group (73 slugs) and the shamoperated group (63 slugs) following the memory retention test on the same day. Here, the diluted powder mixture was laid in a $180^{\circ}$ arc with a $6-\mathrm{cm}$ radius from the marked point, and the time each slug took to reach the powder mixture was recorded. The odor sensibility test using garlic homogenate was not performed in Experiment II.

\section{Monitoring the behavior of the slugs}

The slugs' behavior was traced by analyzing the video movies recorded by a digital video camera recorder (Handycam, SONY) from upright above $(70 \mathrm{~cm})$. The slugs were starved for $8 \mathrm{~d}$ after the PC ablation (eight slugs) or sham operation (seven slugs). A slug was gently placed on a glass plate at the center of a circle (5-cm radius), whose halves consisted of everyday food/onion homogenate or everyday food/garlic homogenate. The initial direction of the head was gently adjusted toward the border of the two odor sources. The video recording was continued until the slug reached either odor source. In the off-line analysis, the head position of the slug was plotted every $5 \mathrm{sec}$, and the dots were connected by a line.

\section{Section staining and image analysis}

One day after all the behavioral tests of Experiment I were completed, a random sample of slugs that had avoided the carrot juice (five slugs) and slugs that had not avoided the carrot juice (11 slugs) from the PC ablation group were chosen. These slugs were anesthetized by injection of $\sim 500 \mu \mathrm{L}$ of ice-cold $\mathrm{Mg}^{2+}$ buffer into the body cavity, and the whole CNS was dissected out. The CNS was frozen in Tissue-Tek optimal cutting temperature (OCT) compound (Sakura) using liquid nitrogen. Cryostat sections (10 $\mu \mathrm{m}$ thick) were cut and mounted onto glass slides coated with Vectabond (Vector Laboratories). The sections were dried for 30 min at room temperature followed by staining using $0.2 \%$ toluidine blue solution, and cover glasses were mounted using Eukitt (O. Kindler). The images of the stained sections were obtained using a light microscope IX-70 (Olympus) with an attached CCD camera DP70 (Olympus). The area of the residual PC was defined as consisting of intensely stained small cell bodies within a region circumscribed by the sheath of the PC and a line connecting the two constricted portions at the base of the PC. This area $\left(\mathrm{mm}^{2}\right)$ was calculated using computer software Canvas 5 (Deneba) by an experimenter who was not informed of the slug's avoidance behavior during the memory retention test.

\section{Statistical analysis}

All data were expressed as mean \pm SEM. Differences between groups were examined for statistical significance using $\chi^{2}$ test and the two-tailed $t$-test. A $P$-value $<0.05$ denoted the presence of a statistically significant difference.

\section{Acknowledgments}

We thank Syohei Hoshino for his assistance in data analysis. This study was supported by Grants-in-Aid for Scientific Research from the Japanese Ministry of Education, Culture, Science, Sports, and Technology (nos. 15390010, 15790025, 15790036, 16659011).

\section{References}

Balaban, P.M., Vehovszky, A., Maksimova, O.A., and Zakharov, I.S. 1987. Effect of 5,7-dihydroxytryptamine on the food-aversive conditioning in the snail Helix lucorum L. Brain Res. 404: 201-210.

Chang, J.J. and Gelperin, A. 1980. Rapid taste-aversion learning by an isolated molluscan central nervous system. Proc. Natl. Acad. Sci. 77: 6204-6206.

Chase, R. and Tolloczko, B. 1989. Interganglionic dendrites constitute an output pathway from the procerebrum of the snail Achatina fulica. J. Comp. Neurol. 283: 143-152.

. 1993. Tracing neural pathways in snail olfaction: From the tip of the tentacles to the brain and beyond. Microsc. Res. Tech. 24: $214-230$.

Davis, R.L. 2004. Olfactory learning. Neuron 44: 31-48.

de Belle, J.S. and Heisenberg, M. 1994. Associative odor learning in Drosophila abolished by chemical ablation of mushroom bodies. Science 263: 692-695.

Dubnau, J., Grady, L., Kitamoto, T., and Tully, T. 2001. Disruption of neurotransmission in Drosophila mushroom body blocks retrieval but not acquisition of memory. Nature 411: 476-480.

Ermentrout, B., Wang, J.W., Flores, J., and Gelperin, A. 2001. Model for olfactory discrimination and learning in Limax procerebrum incorporating oscillatory dynamics and wave propagation. $J$. Neurophysiol. 85: 1444-1452.

Gelperin, A. 1975. Rapid food-aversion learning by a terrestrial mollusk. Science 189: 567-570.

. 1999. Oscillatory dynamics and information processing in olfactory systems. J. Exp. Biol. 202: 1855-1864.

Gelperin, A. and Tank, D.W. 1990. Odour-modulated collective network oscillations of olfactory interneurons in a terrestrial mollusc. Nature 345: $437-440$.

Gervais, R., Kleinfeld, D., Delaney, K.R., and Gelperin, A. 1996. Central and reflex neuronal responses elicited by odor in a terrestrial mollusk. J. Neurophysiol. 76: 1327-1339.

Hawkins, R.D., Kandel, E.R., and Siegelbaum, S.A. 1993. Learning to modulate transmitter release: Themes and variations in synaptic plasticity. Annu. Rev. Neurosci. 16: 625-665.

Inoue, T., Inokuma, Y., Watanabe, S., and Kirino, Y. 2004. In vitro study of odor-evoked behavior in a terrestrial mollusk. J. Neurophysiol. 91: $372-381$.

Inoue, T., Murakami, M., Watanabe, S., Inokuma, Y., and Kirino, Y. 2006. In vitro odor-aversion conditioning in a terrestrial mollusk. $J$. Neurophysiol. 95: 3898-3903.

Ito, I., Kimura, T., and Ito, E. 2001. Odor responses and spontaneous oscillatory activity in tentacular nerves of the terrestrial slug, Limax marginatus. Neurosci. Lett. 304: 145-148.

Izquierdo, I., Quillfeldt, J.A., Zanatta, M.S., Quevedo, J., Schaeffer, E., Schmitz, P.K., and Medina, J.H. 1997. Sequential role of hippocampus and amygdala, entorhinal cortex and parietal cortex in formation and retrieval of memory for inhibitory avoidance in rats. Eur. J. Neurosci. 9: 786-793.

Kimura, T., Suzuki, H., Kono, E., and Sekiguchi, T. 1998a. Mapping of interneurons that contribute to food aversive conditioning in the slug brain. Learn. Mem. 4: 376-388.

Kimura, T., Toda, S., Sekiguchi, T., and Kirino, Y. 1998b. Behavioral modulation induced by food odor aversive conditioning and its influence on the olfactory responses of an oscillatory brain network in the slug Limax marginatus. Learn. Mem. 4: 365-375.

Matsuo, R., Hitomi, T., Watanabe, S., and Kirino, Y. 2002. Delayed-onset amnesia caused by protein synthesis inhibition in odor-taste associative memory of the terrestrial slug Limax valentianus. Neurosci. Lett. 334: 201-205.

Mayford, M., Bach, M.E., Huang, Y.Y., Wang, L., Hawkins, R.D., and Kandel, E.R. 1996. Control of memory formation through regulated expression of a CaMKII transgene. Science 274: 1678-1683.

McGuire, S.E., Le, P.T., and Davis, R.L. 2001. The role of Drosophila mushroom body in olfactory memory. Science 293: 1330-1333.

Murakami, M., Watanabe, S., Inoue, T., and Kirino, Y. 2003. Odor-evoked responses in the olfactory center neurons in the 


\section{Kasai et al.}

terrestrial slug. J. Neurobiol. 58: 369-378.

Nakaya, T., Kawahara, S., Watanabe, S., Lee, D.S., Suzuki, T., and Kirino, Y. 2001. Identification and expression of a novel gene in odour-taste associative learning in the terrestrial slug. Genes Cells 6: 43-56.

Otto, T., Cousens, G., and Herzog, C. 2000.Behavioral and neuropsychological foundations of olfactory fear conditioning. Behav. Brain Res. 110: 119-128.

Sahley, C.L. 1990. The behavioral analysis of associative learning in the terrestrial mollusc Limax maximus: The importance of inter-event relationships. In Connectionist modeling and brain: The developing interface function (eds. S. Hanson and C. Olson), pp. 36-73. MIT Press, Cambridge, MA.

Sahley, C.L., Gelperin, A., and Rudy, J.W. 1981. One-trial associative learning modifies food odor preferences of a terrestrial mollusk. Proc.
Natl. Acad. Sci. 78: 640-642.

Sakura, M., Kabetani, M., Watanabe, S., and Kirino, Y. 2004. Impairment of olfactory discrimination by blockade of nitric oxide activity in the terrestrial slug Limax valentianus. Neurosci. Lett. 370: 257-261.

Teyke, T. 1996. Nitric oxide, but not serotonin, is involved in acquisition of food-attraction conditioning in the snail Helix pomatia. Neurosci. Lett. 206: 29-32.

Teyke, T. and Gelperin, A. 1999. Olfactory oscillations augment odor discrimination not odor identification by Limax CNS. Neuroreport 10: $1061-1068$

Received March 20, 2006; accepted in revised form May 2, 2006. 


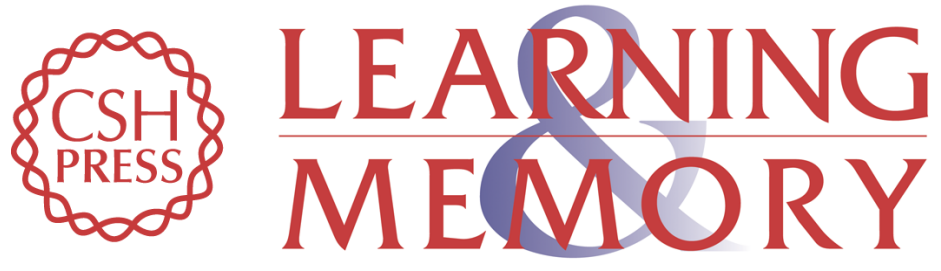

\section{The procerebrum is necessary for odor-aversion learning in the terrestrial slug Limax valentianus}

Yoko Kasai, Satoshi Watanabe, Yutaka Kirino, et al.

Learn. Mem. 2006, 13:

Access the most recent version at doi:10.1101//m.257606

References This article cites 28 articles, 9 of which can be accessed free at:

http://learnmem.cshlp.org/content/13/4/482.full.html\#ref-list-1

License

Email Alerting Receive free email alerts when new articles cite this article - sign up in the box at the Service top right corner of the article or click here. 